\title{
Picosecond Laser Beam Interference Ablation of Thin Metal Films on Glass Substrate
}

\author{
Eglè Molotokaite ${ }^{1}$, Mindaugas Gedvilas ${ }^{1}$, Gediminas Račiukaitis ${ }^{1}$, Valdas Girdauskas ${ }^{2}$ \\ ${ }^{l}$ Laboratory for Applied Research, Institute of Physics, Savanoriu Ave. 231, LT-02300 Vilnius, \\ Lithuania \\ E-mail: graciukaitis@ar.fi.lt \\ ${ }^{2}$ Physics Department, Vytautas Magnus University, Vileikos str. 8, LT-44404 Kaunas, Lithuania
}

\begin{abstract}
Interfering laser beams with the high pulse energy provide an opportunity of direct laser patterning of materials in a predetermined way. This study reports results of the laser beam interference ablation of thin metal films on the glass substrate irradiated with the picosecond laser. Gratings and grids consisting of metal wires of several hundred nanometers were produced in a chromium film. A matrix of nanodots was obtained after irradiation of a aluminum film. The period of structures caused by interference was close to $2 \mu \mathrm{m}$.

DOI: $10.2961 / \mathrm{jlmn} .2010 .01 .0016$
\end{abstract}

Keywords: interference, metal film, laser ablation, picosecond laser, periodical

\section{Introduction}

Properties of the surface highly depend on its relief. The best example is a lotus leaf with the "self-cleaning", hydrofobic feature. Micro- and nano-structuring with specific patterns and at localized places is useful to control wettability, lubrication and other properties of the surface. Artificial structures borrowed from the nature are applied in automotive industry as well as in photonics and biomedical research.

A laser beam may be applied to make diverse structures on most technical and biological surfaces. Laser direct writing (LDW) is a flexible method with spatial resolution limited by a spot size. Performance of LWD for real applications on large areas is too low when fine structures should be produced [1]. Laser induced periodical surface structures, ripples, [2] are useful to control surface absorbivity [3], however, it is difficult to create a desirable shape.

Laser patterning using interference of several beams is the technique capable of producing the sub-wavelength features not limited by a beam spot size and it is an effective method of forming two-dimensional (2D) and threedimensional (3D) structures [4]. The periodical structure can be controlled by changing an incidence angle of the beams, radiation wavelength, phase difference between the beams, polarization and energy. 1D and 2D structures were produced by combining several laser beams [5]. Holographic lithography by combining a few beams of a femtosecond laser and the controllable phase shift between them was used for formation of periodic structures in photopolymers [6].

Different techniques are applied to produce the interfering field of laser radiation. In the Lloyd's schema, part of the expanded beam is reflected from a tilted mirror and it interferes with the incident light on a sample surface. The period of the patterns can be adjusted by an angle between the mirror and samples [7]. The schema is simple, but it can be used with the cw or long pulse lasers. Compensation of temporal delay between separate beams is required to get interference with short laser pulses when the laser beam is split using partially reflecting beam-splitters [8,9].

For ultra-short laser pulses $(<10 \mathrm{ps})$ diffractive optical element (DOE) is preferable instead of mirrors, as DOE inclines the wave front in such a way that it remains perpendicular to the common optical axis and an overlap of the beams is ensured over the whole beam diameter. The optical path for all beams is exactly the same, and the period of the structure is independent of the wavelength, as it is defined by the DOE period [10]. DOE has recently become popular for splitting the laser beam into the desired (more than two) number of beams as well. Additional optics is applied to combine those beams to produce interference patterns [6].

The interfering laser beam with a high pulse energy was applied to direct laser ablation in bulk metals $[11,12]$ and metal films $[13,14]$. The optical absorbance of the metallic surface was enhanced by using the interferometric laser surface patterning [15].

In this work, the high pulse energy picosecond laser was applied to produce interference patterns in thin metallic films by direct ablation. The laser beam interference ablation (LBIA) was tested as a method of laser patterning over large areas.

\section{Experimental setup and procedures}

The picosecond laser PL2241 (Ekspla Ltd.) with a high pulse energy $(1 \mathrm{~mJ})$ was used in the experiments. The pulse duration at FWHM was $25 \mathrm{ps}$. The laser generated the $1064 \mathrm{~nm}$ radiation at the repetition rate of $250 \mathrm{~Hz}$. The laser was selected because of the high pulse energy, and the laser fluence above the ablation threshold was maintained over a large focal area. The attenuator made of a thin halfwave phase plate and a thin film polarizer was used to control the laser pulse energy. The laser beam was split into two beams at an angle of 10 degrees by the diffractive optical element (DOE, Holo/Or Ltd.) with a period of $12 \mu \mathrm{m}$. The diffraction efficiency to \pm 1 order maxima was $40 \%$ 
each with $2.5 \%$ remaining in a zero-order beam. The trap was used to block the zero order maximum of the diffracted beam, because it was found that even such low intensity in the beam significantly affected the period of interference modulation. The piezoelectric 3-axis positioning system PIHera (Physical Instruments) with the movement range of $0.5 \mathrm{~mm}$ in $\boldsymbol{x y}$ and $0.25 \mathrm{~mm}$ in $\boldsymbol{z}$ directions was used for precise focusing of the beam and scanning of the sample. Experimental setup is shown in Fig.1.

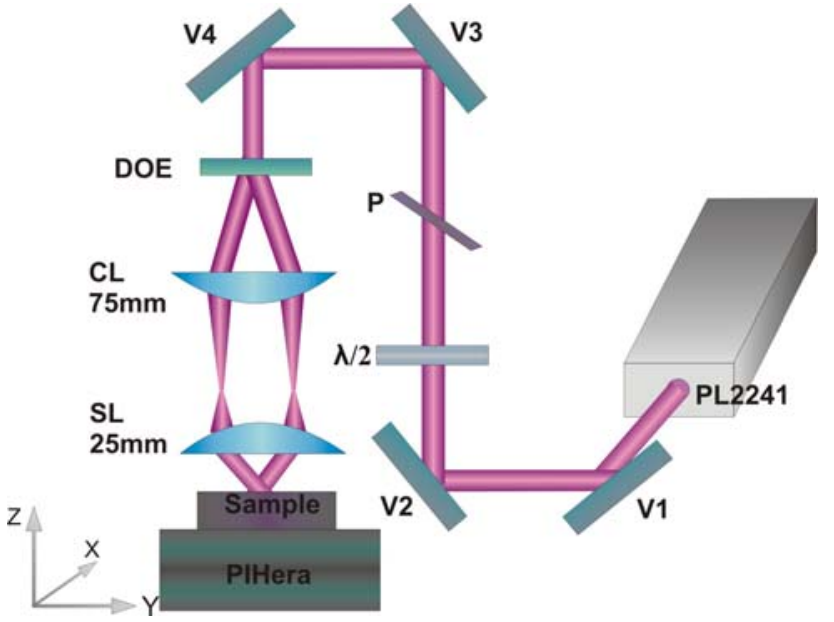

Fig. 1 Setup for the laser beam interference ablation experiments with two beams and 4F imaging system. PL2241 - laser, $\lambda / 2$ - half-wave phase plate; $\mathrm{P}$ - thin film polarizer; DOE - diffractive optical element; CL - cylindrical lens; SL - spherical lens; PIHera-piezoelectric 3-axis positioning system.

The image of the DOE was translated to the workpiece surface by two lenses arranged in the $4 \mathrm{~F}$ system. The first lens with the focal length of $\boldsymbol{F}_{\boldsymbol{1}}$ was placed at that distance from DOE, and both beams propagated in parallel. The second lens was added at a distance equal to the sum of both focal lengths $\boldsymbol{F}_{\boldsymbol{1}}+\boldsymbol{F}_{2}$. Both splitted beams interacted beyond the second lens, creating the interference patterns in the intersection area.

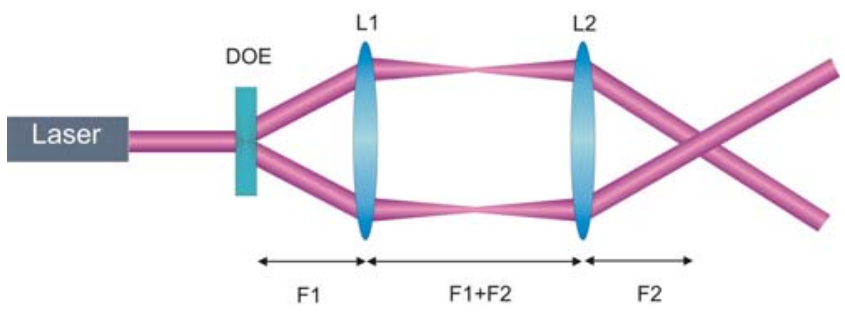

Fig. 2 The optical imaging system with two lenses in $4 \mathrm{~F}$ arrangement.

The 4F-lens image translation system made of a cylindrical (CL) lens and a spherical (SL) lens was used to make interference pattern on the sample. One cylindrical lens was used instead of a spherical one to focus the beam in one direction in order to get a higher energy density on a workpiece. The energy density in the elliptical spot modulated by the interference was given by:

$$
F(x, y)=F_{0} e^{-\frac{2 x^{2}}{w_{0 x}^{2}}-\frac{2 y^{2}}{w_{0 y}^{2}}}\left[1+\cos \left(2 \pi x / \Lambda_{2}\right)\right],
$$

where $\boldsymbol{x}, \boldsymbol{y}$ are coordinates in the workpiece plane, $\boldsymbol{F}_{\boldsymbol{0}}$ is the energy density in the center of the Gaussian beam. The spot waists $\boldsymbol{w}_{\boldsymbol{0}}$ and $\boldsymbol{w}_{\boldsymbol{y}}$ in the $\boldsymbol{x}$ and $\boldsymbol{y}$ directions, respectively, $\left(1 / \mathrm{e}^{2}\right.$ level) were measured by using a simple technique [16]. $\boldsymbol{\Lambda}_{2}$ is the interference modulation period. The energy density in the center of the beam can be estimated from:

$$
F_{0}=\frac{2 E_{\mathrm{p}}}{\pi w_{0 x} w_{0 y}}
$$

$\boldsymbol{E}_{\boldsymbol{p}}$ is the whole pulse energy emitted by the laser. Due to interference, the fluence was equal to $2 \mathrm{~F}_{0}$ at the interference maximum and zero at the minimum. The modulation period in the workpiece plane due to interference can be written as:

$$
\Lambda_{2}=\frac{\Lambda_{1}}{2} \frac{F_{2}}{F_{1}}
$$

$\boldsymbol{F}_{1}$ and $\boldsymbol{F}_{2}$ are the focal length of the CL and SL lenses in the $4 \mathrm{~F}$ imaging system, $\boldsymbol{\Lambda}_{\boldsymbol{l}}$ is the period of the DOE. The numerical calculations were performed in order to determine sensitivity of the interference patterns to the alignment of beams. Deviation from the exact position led to the reduction in intensity maxima and the modulation depth. Modulation disappeared when a workpiece was displaced by at least $0.5 \Delta z z_{R}\left(z_{R}-\right.$ Raleigh length) from the focal plane of the second lens.

The ablation threshold of complete removal of the chromium thin film from the glass substrate was measured by using a simple technique [16] based on the regression method by measuring ablation diameters at different fluences. The ablation (film removal) threshold $\boldsymbol{F}_{\boldsymbol{t} \boldsymbol{h}}$ was found to be equal to $1.4 \mathrm{~J} / \mathrm{cm}^{2}$ (incident energy) for the chromium film, and the spot size was as large as $300 \times 7 \mu \mathrm{m}^{2}$.

\section{Irradiation of chromium film by the interfering la- ser beam}

\subsection{Single pulse ablation}

A thin chromium film with the thickness of $100 \mathrm{~nm}$ on the glass substrate was used in the experiment. An elliptical spot with intensity modulation due to interference along the long axis was applied on the sample. The metal was ablated at the interference maxima. The chromium film ablated with a single laser pulse is shown in Fig.3.

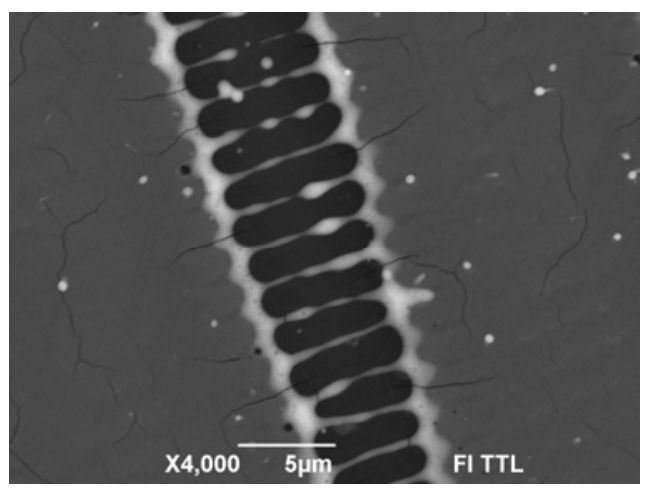

Fig. 3 The scanning electron microscope (SEM) image of resolidified chromium grating on a glass substrate made by a single pulse irradiation with two interfering laser beams. The black area presents openings in the chromium film. Dark grey area is an initial chromium film. The white area is re-solidified chromium. 
The period of the re-solidified chromium grating was $2 \mu \mathrm{m}$ and it was in agreement with the period from calculations by using equation (3).

\subsection{D periodical grating formation}

The regular 1D grating on a thin chromium film was produced over a large area by scanning the interfering beam through the workpiece along the interference lines (yaxis). The resulting picture is shown in Fig. 4.

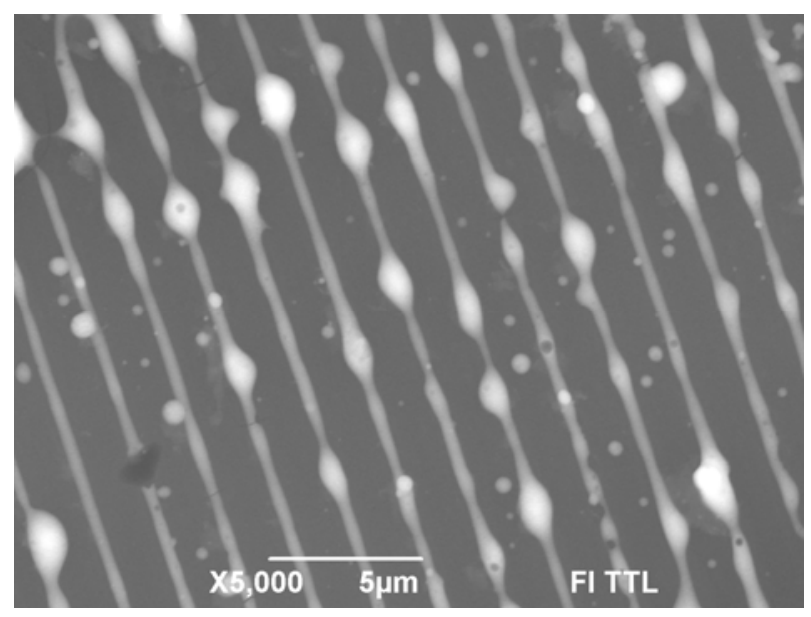

Fig. 4 SEM image of the re-solidified chromium grating on a glass substrate made by the laser beam interference ablation.

Metal was ablated at the interference maxima by a single laser pulse. The sample was shifted along interference lines (y-axis) by a distance shorter than the spot dimension $(7 \mu \mathrm{m})$ to ensure overlapping of laser pulses. The grating was increasing by the line length with every subsequent laser pulse. The submicron wires of chromium were developed from the metal film left on the glass substrate after ablation. The period between chromium wires was $2 \mu \mathrm{m}$. The typical width of chromium wires was $0.2-0.5 \mu \mathrm{m}$. Droplets of metal remained on the wires. The droplets were formed from the redundant molten metal. It was possible to arrange them regularly (see insert in Fig.5a) by controlling the translation speed in $\boldsymbol{y}$ direction and the pulse energy. The speed was responsible for spatial overlapping of laser pulses.

\subsection{D periodical grating formation}

The sample was fixed on 3-axis piezo-stage in order to get structures over a large area. The positioning system was able to move $500 \mu \mathrm{m}$ in $\boldsymbol{x}$ and $\boldsymbol{y}$ directions and $250 \mu \mathrm{m}$ in the $z$ direction with the minimal programmable step of $10 \mathrm{~nm}$. The translation speed and laser pulse energy were varied in a wide range. Energy density was varied in a wide range starting below the ablation threshold up to the high enough one to ensure complete removal of the film. The spot was scanned in the direction perpendicular to the long axis of an elliptical spot. The scanning speed in the $y$ direction (vertical in Fig. 5-8) was changed to perform laser patterning at high pulse overlapping as well as with separate laser pulses. However, high quality periodical structures, stable over the whole scanning field, were formed when the pulse energy was $70-80 \mu \mathrm{J}$ and the scanning speed was in the range of $550-1000 \mu \mathrm{m} / \mathrm{s}$. In other cases, the chromium film was evaporated completely or the glass substrate was damaged.

It was found that the pulse energy of $77 \mu \mathrm{J}$ was sufficient to evaporate the $\mathrm{Cr}$ film by a single shot. However, the ridges around the evaporated area were always formed from melt. Therefore, three pulses of the same energy were required to remove the metal of the elevated thickness. The 1D-periodical grating was formed when the scanning speed was reduced down to $600 \mu \mathrm{m} / \mathrm{s}$, which corresponded to the shift between laser pulses equal to $2.4 \mu \mathrm{m}$ (laser repetition rate $250 \mathrm{~Hz}$ ). In this case laser fluence was sufficient to remove the ridges formed by the previous laser pulse. At the same time, the laser fluence was not high enough to damage the glass substrate (Fig. 5).

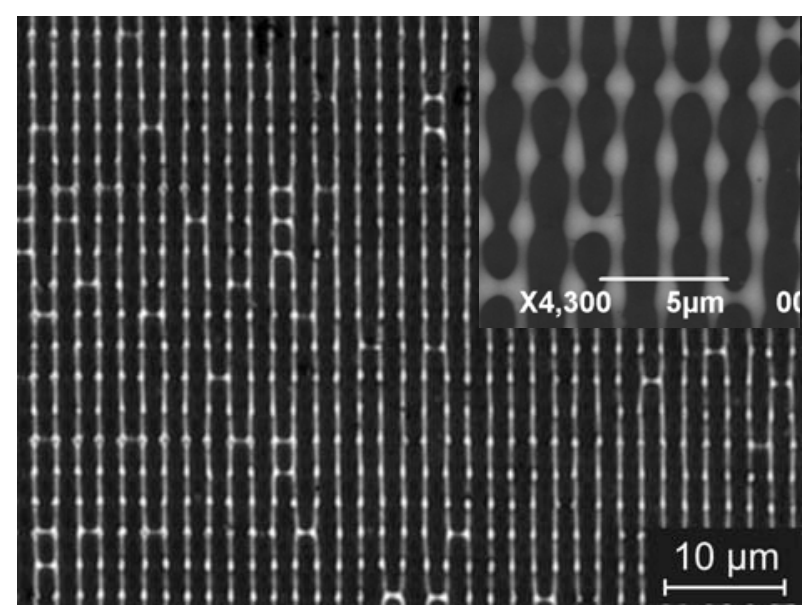

(a)

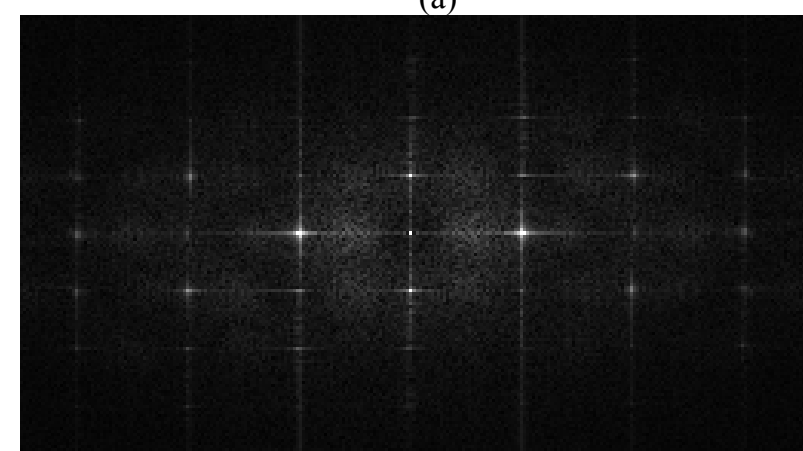

(b)

Fig. 5 Optical microscope image of the 2D re-solidified grating with the period of $2 \mu \mathrm{m} \times 3 \mu \mathrm{m}$ on the glass substrate made by the laser beam interference ablation: a) the bright-field microscopy; insert - SEM image of re-solidified submicron wires on a glass substrate made with pulse energy of $77 \mu \mathrm{J}$; b) Fast Fourier transformation (FFT) of the transmitted light. Pulse energy: $80 \mu \mathrm{J}$; translation speed: $600 \mu \mathrm{m} / \mathrm{s}$.

The Fast Fourier transformation in Fig.5b shows main periods and harmonics in the feature separation over the chromium film irradiated with the laser. Periodicity was maintained with high precision.

The different situation occurred when the scanning speed was increased to $750 \mu \mathrm{m} / \mathrm{s}$. The shift between laser pulses then was $3 \mu \mathrm{m}$. The ridge produced by the previous laser pulse could not be removed with the next pulse. Therefore, the 2D-net type periodical structure with the period of $3 \mu \mathrm{m}$ along the $\boldsymbol{y}$ direction and the period of $\sim 2 \mu \mathrm{m}$ along the $\boldsymbol{x}$ direction due to the laser interference was fabricated (Fig. 6a). Vertical lines are due to interference, while horizontal lines are remains of unablated metal. 
The area of the periodical structure formation was limited by the translation range of the piezoelectric motors.

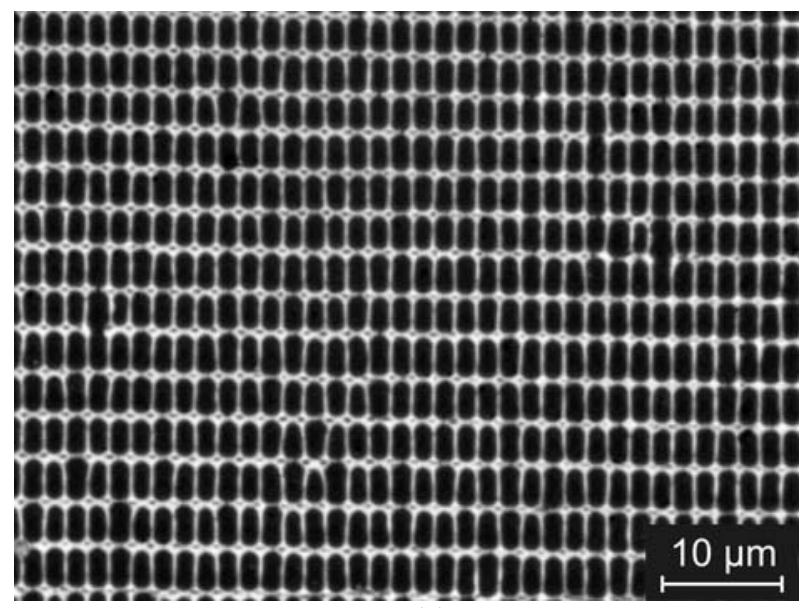

(a)

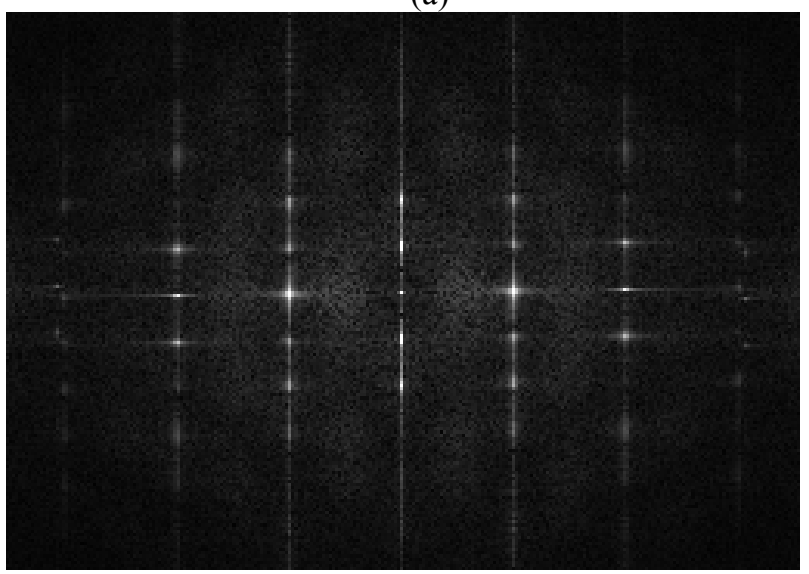

(b)

Fig. 6 Optical microscope image of the 2D re-solidified grating with the period of $2 \mu \mathrm{m} \times 3 \mu \mathrm{m}$ on the glass substrate made by the laser beam interference ablation. (a) bright-field microscopy; (b) FFT of the transmitted light. Pulse energy: $80 \mu \mathrm{J}$; translation speed: $750 \mu \mathrm{m} / \mathrm{s}$.

The grating shows increased diffraction in the $\boldsymbol{y}$ direction (Fig. 6b) compared to the previous picture (Fig.5b) due to periodicity formed by boundaries between the singlepulse ablated areas.

By increasing the scanning speed the period of the structure increased in the $\boldsymbol{y}$ direction. When the speed was higher than $1000 \mu \mathrm{m} / \mathrm{s}$, the structures became unstable over a large area due to variation in the translation performance of the translation stages. The areas ablated with single shots did not overlap when the speed was increased above $1500 \mu \mathrm{m} / \mathrm{s}$ as the shift between pulses exceeded the beam diameter along the $\boldsymbol{y}$ axis and separate ablated holes were produced.

\section{Results on LBIA in other metal films}

Similar laser beam interference ablation tests were performed with different metals on the glass substrate: aluminum, gold, silver, aluminum and copper. The thickness of the metal foil on the glass substrate was $100 \mathrm{~nm}$ for all metal films.

In aluminum, no ridges were formed on rims of the ablated area. The metal was completely removed when the laser fluence was above the ablation threshold. Therefore, the only structure we succeeded to form in the metal was a matrix of sub-micron dots of aluminum on irradiation boundary of two not-overlapping laser spots modulated by the interference (Fig. 6).

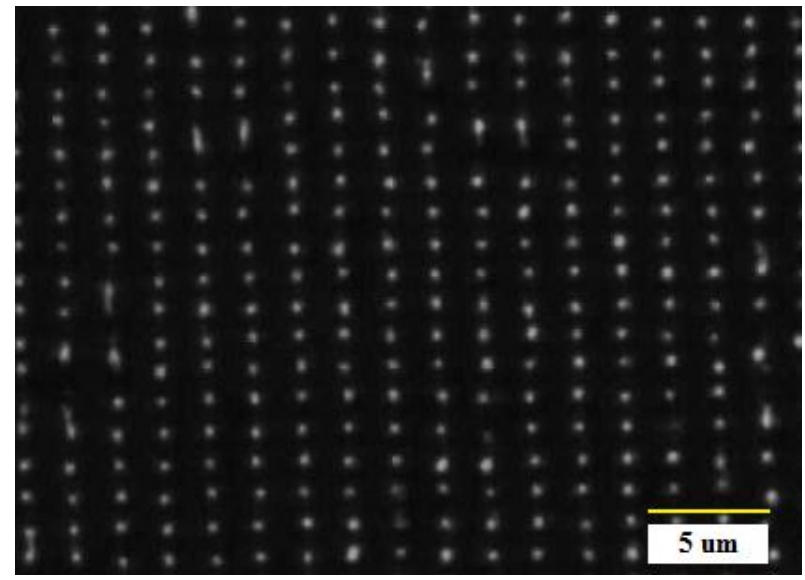

Fig. 7 Matrix of nano-dots remaining of aluminum film after repetitive irradiation of the aluminum film with the interfering laser beam. Pulse energy: $50 \mu \mathrm{J}$; translation speed: $750 \mu \mathrm{m} / \mathrm{s}$.

When the pulse energy was reduced to $15 \mu \mathrm{J}$ keeping the same scanning speed $(v=750 \mu \mathrm{m} / \mathrm{s})$, periodically spaced Al triangles were formed instead of circular dots.

The gold film was tested in a wide range of lasertranslation parameters. The pulse energy was varied from $70 \mu \mathrm{J}$ to $200 \mu \mathrm{J}$, and the translation speed - from $7 \mu \mathrm{m} / \mathrm{s}$ to $750 \mu \mathrm{m} / \mathrm{s}$. Regular structures were not formed in gold, only partially developed periodical features were found in the a very narrow range of process parameters (Fig. 8).

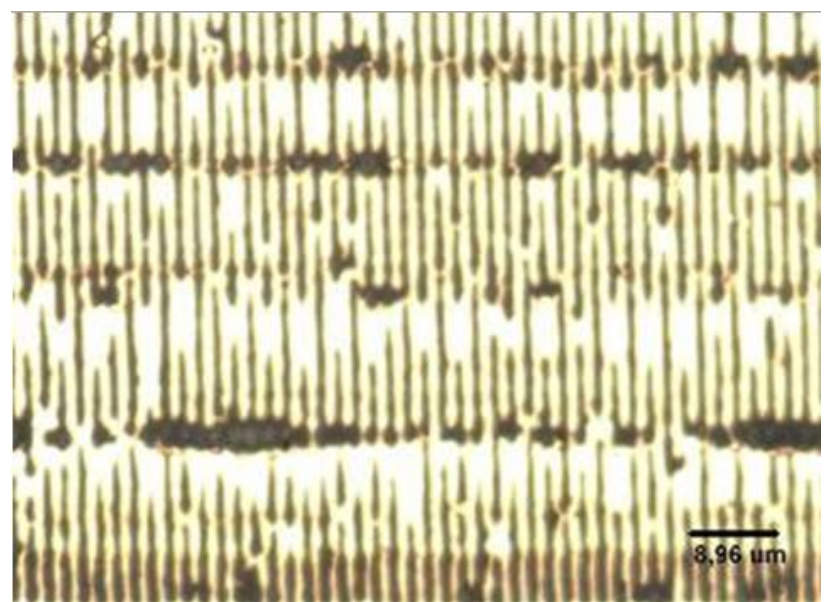

Fig. 8 Partially developed periodical structures in the gold film. Small variations in the process parameters (pulse energy, translation speed, etc.) led to the abruptive 1D structure. Pulse energy: $80 \mu \mathrm{J}$; translation speed: $20 \mu \mathrm{m} / \mathrm{s}$.

The silver film was tested when the pulse energy was varied from $10 \mu \mathrm{J}$ to $70 \mu \mathrm{J}$, and the translation speed was from $50 \mu \mathrm{m} / \mathrm{s}$ to $750 \mu \mathrm{m} / \mathrm{s}$. The copper film was tested when pulse energy was varied from $200 \mu \mathrm{J}$ to $350 \mu \mathrm{J}$, and the translation speed was from $50 \mu \mathrm{m} / \mathrm{s}$ to $1200 \mu \mathrm{m} / \mathrm{s}$. In both cases, no regular periodical structures were observed. Laser energy ablated completely the film at a higher irradiation dose, or no effect on the film structure was ob- 
served at lower irradiation from the examined range of the process parameters.

\section{Discussion}

The structures were formed in all cases when laser fluence at interference maxima was above the ablation threshold (and the film removal threshold), estimated without interference. In that situation, evaporation of metal was the main driving force in creation of periodical openings in metal films.

Even using the identical periodical interference field to irradiate the metal films, they behaved differently. Transformations in the thin metal film appeared after laser irradiation due to the coupled energy. Melting and vaporization of the metal took place when the energy density was high enough to increase the film temperature. Interference of the laser beam intensity along the long axis of the elliptical spot in the focal plane led to periodical variation of the coupled energy in the metallic film.

The finite element simulation of temperature profile dynamics was carried out to calculate variation of the surface temperature over a single interference period depending on the time spent after the irradiation started. The pulse duration of the used laser was 25 ps. That moment corresponded to the highest temperature reached at the interference maximum. The Multiphysics software package (COMSOL) was used for simulation. The intensity absorbed by the metals was kept the same $\left(I_{0}\right.$ absorbed $=$ $1.2 \times 10^{13} \mathrm{~W} / \mathrm{m}^{2}$, which corresponds to fluence $F_{0}$ absorbed $=$ $0.03 \mathrm{~J} / \mathrm{cm}^{2}$ ) to have possibility to compare the behavior of different materials at that level of excitation. The absorption coefficient was assumed to be as high as $\alpha=7 \times 10^{5} \mathrm{~cm}^{-1}$, which corresponded to the absorption depth of $1 / \alpha=14 \mathrm{~nm}$, when the film thickness was $100 \mathrm{~nm}$. The parameters typical for the metals with high reflectivity were selected, and no variation in surface reflection depending on the temperature was considered. Results of simulations are shown in Fig. 9.

Even at that low irradiation intensity, the temperature of metallic films increased above the evaporation point in a wide area surrounding the interference maximum for all the metals during the laser pulse duration. Thermal conductivity forced the heat diffusion toward the interference minima. Surface tension forces in molten metal facilitated formation of metal wires at interference minima until solidification took place. They were responsible for the final shape (cross-section) of metal wires remained on the glass substrate, but they were not included into modeling.

A similar simulation for bulk metals was performed in [12]. The temperature profiles as well as the quantities of molten and vaporized material as a function of the laser fluence value were estimated. The duration of the structuring process (molten phase) was found to be as long as $600 \mathrm{~ns}$ according to both experimental measurements and the numerical calculations.

According to our simulation, in case of aluminum, the metal film was melted even at the interference minima in the time span exceeding $2.5 \mathrm{~ns}$. The gold film remained solid close to the minima and evaporation was reduced due to a higher evaporation temperature. Due to the low thermal conductivity of chromium, the maximum temperature was high and evaporation was intensive at the interference maxima for a short time period. However, the temperature at the interference minima remained significantly lower than the melting point. This prevented the material removal after irradiation in a wide range of irradiation doses. Therefore, the structure of the interfering laser field was easily transferred to the metal film in case of chromium.

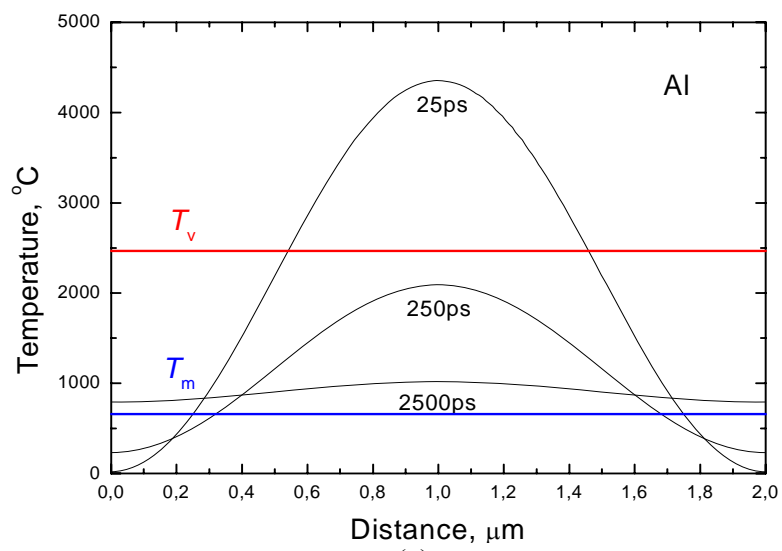

(a)

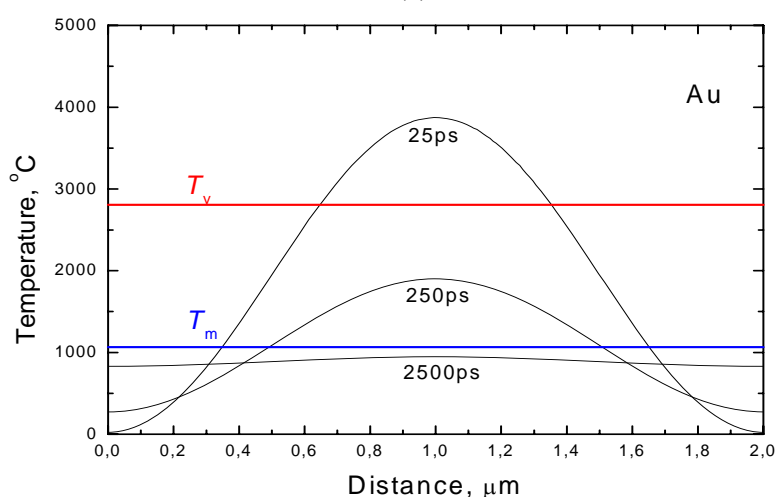

(b)

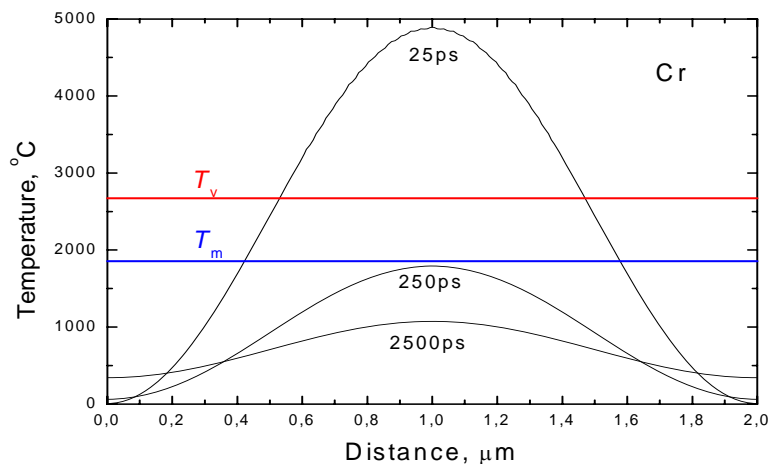

(c)

Fig. 9 Simulation of dynamics in surface temperature distribution in aluminum (a), gold (a) and chromium (c) films irradiated with the interfering laser beam (one longitudinal period). Laser pulse duration: $25 \mathrm{ps}$. Time marked near the curves is counted from the laser pulse start. Melting and evaporation temperatures are shown.

Tendency of chromium to create stable periodical structures was observed by homogeneous linear laser irradiation $[17,18,19]$. The periodical ripple gratings formed by selforganization in the chromium thin film on the glass substrate are similar to the gratings created here by the laser beam interference. But, there are some differences: the period in ripples was gradually changed by controlling the laser fluence or a shift between pulses [17]. The period in the interference grating can be set to only specific values 
by changing the magnification in the $4 \mathrm{~F}$ imaging system. The width of chromium wires in ripples is roughly a half of the grating period, in contrast to the wires made by the interference. The width of wires can be achieved much thinner than the period of grating in this case.

Diverse behavior of the films depending on the metal, the shift between pulses and laser fluence was observed in [20] at homogeneous line irradiation. In chromium, the regular structures were developed in a quite wide range of processing parameters. In gold, three kinds of ripples were developed in a narrow processing window. Aluminum, copper and silver did not show a regular structure.

The results for various metal films have similarities with our results on the laser beam interference ablation. The combination of physical properties of the metals facilitated partial vaporization of the film and structure formation in molten state due to the thermal gradient and surface tension.

\section{Conclusions}

The interfering laser beam was applied to pattern thin metallic films on the glass substrate. The high-pulse-energy picosecond laser, DOE and two-lens imaging system as well as the sample translation system made it possible to create the structures with a period of $2 \mu \mathrm{m}$ over a large area in the chromium film.

The one-dimensional periodical gratings were produced by using the elliptical laser spot modulated by interference and overlapping laser pulses. The metal wires with the width in a sub-micron range were formed. The twodimensional periodical gratings were produced when laser pulses did not overlap significantly. The period could be controlled in the translation direction in the range of $2.5-$ $5 \mu \mathrm{m}$.

Matrix of nanodots was developed in the aluminum film irradiated with a series of not-overlapping laser beams modulated by interference. Stability of the system was not high enough to develop a regular structure in the gold film. Copper and silver did not show any tendency in regular structure formation using the interfering laser field.

Simulation of thermal distribution revealed that differences in heat conduction of metals as well as other thermal properties were responsible for diverse behavior of chromium, gold and aluminum under their irradiation with the interfering laser beam.

The laser beam interference ablation might be used as a method for large area laser patterning.

\section{Acknowledgments}

The work was supported by the Lithuanian State Science and Studies Foundation under project No B-31/2008.

\section{References}

[1] J. Koch, F. Korte, C. Fallnich, A. Ostendorf and B.N. Chichkov, Opt. Engineer., 44, (2005) 051103.

[2] J. Reif, F. Costache, M. Henyk, S.V. Pandelov, Appl. Surf. Sci., 197/198, (2002) 891.

[3] Z. Huang, J.E. Carey, M. Liu, X. Guo, E. Mazur, J.C. Campbell, Appl. Phys. Lett., 89, (2006) 033506.
[4] T. Kondo, S. Matsuo, S. Juodkazis, H. Misawa, Appl. Phys. Lett., 79, (2001) 725.

[5] N. Tsutsumi, A. Fujihara, K. Nagata, Thin Solid Films, 517, (2008) 1487.

[6] T. Kondo, S. Juodkazis, V. Mizeikis, H. Misawa, Opt. Express, 14, (2006) 7943.

[7] Q. Xie, M.H. Hong, H.L. Tan, G.X. Chen, L.P. Shi, T.C. Chong, J. Alloys and Compounds, 449, (2008) 261.

[8] C. Daniel, F. Mücklich, Z. Liu, Appl. Surf. Sci., 208209, (2003) 317.

[9] A.F. Lasagni, D. Yuan, P. Shao, S. Das, Adv. Eng. Mat., 11, (2009) 20.

[10] A.A. Maznev, T.F. Crimmins, K.A. Nelson, Opt. Lett., 23, (1998) 1378.

[11] A. Lasagni, F. Mücklich, Appl. Surf. Sci., 240, (2005) 214.

[12] A. Lasagni, F. Mücklich, J. Mater. Proc. Techn., 209, (2009) 202.

[13] Y. Nakata, J. Muramoto, T. Okada, M. Maeda, Appl. Phys. Lett., 81, (2002) 4239.

[14] M. Mäder, T. Höche, J.W. Gerlach, R. Böhme, K. Zimmer, B. Rauschenbach, Phys. Stat. Sol. (RRL), 2, (2008) 34.

[15] K. Paivasaari, J.J. Kaakkunen, M. Kuittinen, T. Jaaskelainen, Opt. Express, 15, (2007) 13838.

[16] J.M. Liu, Opt. Lett., 7, (1982) 196.

[17] M. Gedvilas, G. Račiukaitis, K. Regelskis, Appl. Phys. A, 93, (2008) 203.

[18] K. Regelskis, G. Račiukaitis, M. Gedvilas, Appl. Surf. Sci., 253, (2007) 6584.

[19] M. Gedvilas, G. Račiukaitis, K. Regelskis, P. Gečys, J. Laser Micro/Nanoengin., 3, (2008) 58.

[20] M. Gedvilas, B. Voisiat, G. Račiukaitis, K. Regelskis, Appl. Surf. Sci., 255, (2009), available on-line (DOI: 10.1016/j.apsusc.2009.04.099).

(Received : July 21, 2009, Accepted : February 4, 2010) 